CASE REPORT

\title{
Narcolepsy: How common is the tetrad?
}

\section{Shivesh Prakash, Nagarajan Ramakrishnan}

Nithra Instiute of Sleep Sciences, Chennai, India

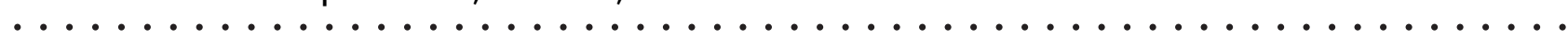

Indian J Sleep Med 2009; 4.2, 68-69
\end{abstract}

\section{Introduction}

The term "narcolepsy" is derived from Greek meaning "seized by somnolence." Gelineau ${ }^{1,2}$ was the first to delineate the syndrome in 1880. More than a century now and still significant number of cases are undiagnosed, misdiagnosed or diagnosed very late adding significantly to its adverse consequences particularly psycho-social morbidity. What is more worrying is the fact that the disease usually affects the adolescent age group which is very vulnerable to its impact on their psychology. One important reason could be an elusive diagnosis as the presentations vary and importantly, the classic 'tetrad' of narcolepsy is very rare particularly in children. A survey of 422 case reports on narcolepsy in literature revealed only two reports of adolescents presenting with the classic tetrad. We recently came across an adolescent who presented with the typical rare tetrad of the disease. The manuscript includes a succinct description of the case presentation and analysis of presentation patterns reported in the literature.

\section{Case Report}

Sixteen year old non-obese adolescent was referred for evaluation for day time hyper-somnolence for one year. History revealed that he usually goes to bed at $11 \mathrm{pm}$ and takes about 5 minutes to fall asleep. Just when he starts sleeping he often complains of some bright indiscernible forms floating around and also complains of feeling paralyzed during that time. He learned that by listening to music he could avoid the feeling of paralysis. He would wake up at 7

\section{Address for Correspondence:}

\section{Dr N. Ramakrishnan}

Nithra Institute of Sleep Sciences

AK 38, $8^{\text {th }}$ Street, $11^{\text {th }}$ Main Road

Annanagar, Chennai 600040

Ph: +9144 43502252 Fax: +91 4426213322

Email: ram@nithra.com am with alarms usually not feeling refreshed. His Epworth Sleeping Score (ESS) was 17. He also complained of sudden feeling of "limbs getting loose" and falling down following bouts of laughter, recovering within couple of minutes and during which his consciousness would be preserved. Past history and physical examination was unremarkable. His lab results including thyroid profile were unimpressive.

An overnight polysomnography revealed that proportion of total sleep time short sleep latency and reduced slow wave sleep and REM sleep, but no evidence of sleep disordered breathing or periodic limb movements. A Multiple Sleep Latency Test (MSLT) study was done as per standard protocol on next day which revealed that average sleep latency was $5 \mathrm{~min}$ and three of the four naps revealed REM sleep (fifth nap was not pursued as per protocol). A Clinical diagnosis of Narcolepsy with Cataplexy was made and patient was started on Modafinil and Fluoxetine (for Cataplexy). Appropriate counseling for the family and patient was given along with advice on non-pharmacological treatment (safety issues, naps etc). Patient reported for follow up after a month and was clinically improving.

\section{Discussion}

The child presented with classical tetrad of narcolepsy along with the typical findings on the polysomnography. This classical presentation is very rare particularly amongst the children. Considering all age groups together, the presentation with this classical tetrad has been reported in as few as $10 \%$ of all cases.

Excessive Daytime Somnolence (EDS) is often the first symptom of narcolepsy ${ }^{3}$, and additional symptoms of the narcolepsy tetrad may develop over time. EDS is present in almost $100 \%$ of cases. Cataplexy is considered to be an excellent discriminating factor for narcolepsy, especially the combination of a history of cataplexy and the incidence of a sleep onset-REM period which has been included as one of the diagnostic criteria. Cataplectic symptoms show 
variation and case reports reveal manifestations which range from mild to severe ${ }^{4}$. The incidence of mild attacks is much more common. Considering all types of cataplectic symptoms together, the prevalence has been found to be about $70 \%$ of cases. Sleep paralysis may terminate spontaneously or on tactile or auditory stimulation which was seen in our case. This symptom may not occur with other symptoms in the narcolepsy tetrad. Reported prevalence varies from 20 to $50 \%$. The types of hypnagogic hallucinations reported in literature also show significant variation and may be visual, auditory, or tactile with visual hallucinations being the commonest. The reported prevalence varies from 20 to $40 \%$. In children, however, the clinical symptoms and polysomnographic findings may be atypical, making it difficult to establish a definite diagnosis. About $30-50 \%$ of cases present without cataplexy. In some cases this feature may take up to 10 years to present. $50-60 \%$ of cases present without hallucinations and $40 \%$ without sleep paralysis.

A survey of 422 case reports on narcolepsy in literature revealed only two reports of adolescents presenting with the classic tetrad. In a case series of 16 adolescents from Pittsburgh School of Medicine ${ }^{5}$ only one had a classic tetrad. The only child with the full tetrad of symptoms had minimal findings on polysomnography, and the child with polysomnographic findings characteristic of narcolepsy presented with excessive daytime sleepiness only. Obesity appeared as an unexpected association in this case series, with 11 of the 16 narcoleptic patients found to be overweight at the time of diagnosis. There was no association between EEG findings and clinical presentation. While 12 had behavioral and emotional disturbances signifying the extent of psycho-social morbidity and need for early diagnosis and appropriate management. Authors recommended that narcolepsy should be considered in the differential diagnosis of any child with EDS. The frequency of such atypical presentations has lead to an emerging consensus that a diagnosis of narcolepsy be considered even in the absence of cataplexy. This may be especially true in the early presentation of cases as the classical tetrad of symptoms tends to evolve over many years and is rarely seen at initial presentation.

Our patient had developed all symptoms at about same time which is again quiet rare. Early identification can also help reduce some of the long-term sequelae. Wise and Gillespie $^{6}$ highlighted the impact, that childhood narcolepsy has, on the loss of normal childhood experiences due to the effect of chronic sleepiness, which may contribute to academic, social and psychological difficulties in later life. A case series reported that $59 \%$ of 85 adults had symptoms before the age of 15 years. Other studies quote similar rates. Challamel et al. ${ }^{7}$ reviewed 97 paediatric cases and found a mean age at onset of 9 years with $8 \%$ aged 5 or less. In Kotagel and Goulding's. ${ }^{8}$ report on 10 children, the mean time interval between the initial onset of hyper somnolence and definitive diagnosis was 49 months. Our case had a delay of one year, but this was in spite of his classical presentation. Given the rarity of classical presentation of narcolepsy in children and importance of early diagnosis in this age group as highlighted by the above evidences, it is unwise to postpone treatment until all four cardinal symptoms are manifest as this can produce significant detrimental comorbid symptomatology.

\section{References}

1. Stanley Finger, "Origins of neuroscience: a history of explorations into brain function". Ch "The process of sleep". OUP 1994, New York. Pg 253

2. Carlos et al. "English Translations Of The First Clinical Reports On Narcolepsy And Cataplexy by Westphal And Gélineau in The Late 19th Century, with Commentary." Clin Sleep Med. 2007 April 15; 3(3): 301-311.

3. Parkes D. "Introduction to the mechanism of action of different treatments of narcolepsy." Sleep. 1994;17(suppl 8):593-596.

4. Moscovitch A, Partinen M, Guilleminault C. "The positive diagnosis of narcolepsy and narcolepsy's borderland. Neurology." 1993;43:55-60

5. Dahl RE, Holttum J, Trubnick L. "A clinical picture of child and adolescent narcolepsy." J Am Acad Child Adolesc Psychiatry. 1994 Jul-Aug;33(6):834-41

6. Wise M, Gillespie S (1996) "Clinical features of childhood narcolepsy." (abs) Sleep Research 25:390

7. Challamel MJ, Mazzola ME, Nevsimalova S, Cannard C, Louis J, Revol M. "Narcolepsy in children." Sleep. 1994 Dec; 17(8 Suppl):S17-20

8. Kotagal S, Goulding P (1996) "The laboratory assessment of daytime sleepiness in childhood." J Clin Neurophysiol 13:208-218 